\title{
Probing the type-II seesaw mechanism through the production of Higgs bosons at a lepton collider
}

\author{
Pankaj Agrawal, ${ }^{1,2, *}$ Manimala Mitra, ${ }^{1,2, \dagger}$ Saurabh Niyogi, ${ }^{3, \$}$ Sujay Shil, ${ }^{1,2,}$ and Michael Spannowsky ${ }^{4, \|}$ \\ ${ }^{1}$ Institute of Physics, Sachivalaya Marg, Bhubaneswar, Odisha 751005, India \\ ${ }^{2}$ Homi Bhabha National Institute, Training School Complex, Anushakti Nagar, Mumbai 400085, India \\ ${ }^{3}$ Gokhale Memorial Girls' College, Harish Mukherjee Road, Kolkata 700020, India \\ ${ }^{4}$ Institute for Particle Physics Phenomenology, Durham University, Durham DH1 3LE, United Kingdom
}

(Received 23 March 2018; published 16 July 2018)

\begin{abstract}
We investigate the production and decays of doubly-charged Higgs bosons for the Type-II seesaw mechanism at an $e^{+} e^{-}$collider with two center of mass energies, $\sqrt{s}=380 \mathrm{GeV}$ and $3 \mathrm{TeV}$, and analyze the fully hadronic final states in detail. Lower mass ranges can be probed during the $380 \mathrm{GeV}$ run of the collider, while high mass ranges, which are beyond the $13 \mathrm{TeV}$ Large Hadron Collider discovery reach, can be probed with $\sqrt{s}=3 \mathrm{TeV}$. For such a heavy Higgs boson, the final decay products are collimated, resulting in fat-jets. We perform a substructure analysis to reduce the background and find that a doublycharged Higgs boson in the mass range 800-1120 GeV can be discovered during the $3 \mathrm{TeV}$ run, with integrated luminosity $\mathcal{L} \sim 95 \mathrm{fb}^{-1}$ of data. For $380 \mathrm{GeV}$ center of mass energy, we find that for the doublycharged Higgs boson in the range $160-172 \mathrm{GeV}$, a $5 \sigma$ significance can be achieved with only integrated luminosity $\mathcal{L} \sim 24 \mathrm{fb}^{-1}$. Therefore, a light Higgs boson can be discovered immediately during the run of a future $e^{+} e^{-}$collider.
\end{abstract}

DOI: $10.1103 /$ PhysRevD.98.015024

\section{INTRODUCTION}

With the discovery of the Higgs boson at the Large Hadron Collider (LHC), we start to develop an understanding of how the standard model (SM) fermion and gauge boson masses are generated in terms of the BroutEnglert-Higgs (BEH) mechanism. However, one of the main puzzles that still remains unclear is the origin of light neutrino masses and mixings. The same BEH mechanism can, in principle be employed to generate Dirac mass of SM neutrinos by extending the SM to include right-handed neutrinos. However, the required large hierarchy of the Yukawa couplings raises uncomfortable questions. A completely different ansatz is that neutrinos are their own antiparticles and hence, their masses have a different origin than the other SM fermions. A tiny eV Majorana neutrino mass can be generated by the seesaw mechanism, where light neutrinos acquire their masses from a lepton

\footnotetext{
*agrawal@iopb.res.in

†manimala@iopb.res.in

\$saurabhphys@gmail.com

$\S$ sujay@iopb.res.in

"michael.spannowsky@durham.ac.uk
}

Published by the American Physical Society under the terms of the Creative Commons Attribution 4.0 International license. Further distribution of this work must maintain attribution to the author(s) and the published article's title, journal citation, and DOI. Funded by SCOAP ${ }^{3}$. number violating (LNV) $d=5$ operator $L L H H / \Lambda[1,2]$. Such operator is not forbidden as the lepton number is only a classical symmetry of the SM, violated by quantum effects.

There are three proposed categories, commonly known as, Type-I, -II, and -III seesaw mechanisms in which the SM is extended by a $S U(2)_{L}$ singlet fermion [3-9], $S U(2)_{L}$ triplet scalar boson [10-13], and $S U(2)_{L}$ triplet fermion [14], respectively. In particular, the second possibility, i.e., where a triplet scalar field with the hypercharge $Y=+2$ is added to the SM, is the simplest model with an extended Higgs sector. The neutral component of the triplet acquires a vacuum expectation value (vev) $v_{\Delta}$, and generates neutrino masses through the Yukawa interactions. Perhaps, the most appealing feature of this model is its minimality. The same Yukawa interaction between the lepton doublet and the triplet scalar field generates Majorana masses for the neutrinos, and also dictates the phenomenology of the charged Higgs bosons.

A number of detailed studies have already been performed for the Hadron colliders like, Tevatron [15] and LHC [15-29] to search for the triplet Higgs scenario. One attractive feature of this model is the presence of the doubly-charged Higgs boson, and its distinguishing decay modes. Depending on the triplet vev, the doubly-charged Higgs boson can decay into same-sign dilepton, samesign gauge bosons, or even via a cascade decay [16-18]. 
The details of the Higgs spectrum have been discussed in [30-32]. For the branching ratios and collider signatures, see [16-20]. The CMS and ATLAS collaborations have searched for the same-sign dilepton final states for all flavors, and constrained the mass of the doubly-charged Higgs as $M_{H^{ \pm \pm}}>820,870 \mathrm{GeV}$ at $95 \%$ C.L. [33,34]. However, this is only relevant for a very tiny vev $v_{\Delta}<10^{-4} \mathrm{GeV}$, where the doubly-charged Higgs boson decays into the same-sign dilepton with $100 \%$ branching ratio. For larger triplet vev such as $v_{\Delta} \gtrsim 0.01 \mathrm{GeV}$, this branching ratio is negligibly small. Therefore, a direct bound on the mass of the $H^{ \pm \pm}$from the same-sign dileptonic final state cannot be obtained. An alternative search where the $H^{ \pm \pm}$is produced in association with two jets (vector boson fusion channel) gives relaxed constraints [35,36]. For $v_{\Delta} \geq 10^{-4} \mathrm{GeV}$, the doubly-charged Higgs boson predominantly decays into same-sign diboson. The collider signatures and the discovery prospect of this scenario have been discussed in [37-39], and [40,41] (see [42] for the discussion on the composite Higgs model and [43] for discussion on flavor violating $\tau$ decays). Previous searches for $H^{ \pm \pm}$in the pair-production channel and their subsequent decays into same-sign leptons at LEP-II has put a constraint $M_{H^{ \pm \pm}}>97.3 \mathrm{GeV}$ at 95\% C.L. [44].

While a number of searches at the LHC are ongoing to experimentally verify the presence of the doubly-charged Higgs boson, in this work we perform a detailed collider analysis to explore the discovery prospects at a future lepton collider. For a large mass of the doubly-charged Higgs boson, the pair-production cross-section at the LHC becomes small. Furthermore, the presence of numerous backgrounds weakens its discovery prospects. Therefore, a lepton collider with a much cleaner environment will be more suitable to search the high mass regime of the doublycharged Higgs boson. In addition, we also exhaust the low mass regime, yet unconstrained by the LHC, and by LEP-II measurements.

We consider the pair-production of the doubly-charged Higgs boson at a lepton collider and its subsequent decays into same-sign gauge bosons. We focus on the hadronic decays of the produced gauge bosons and analyze the multijet final states in detail. As a prototype example, we consider the future $e^{+} e^{-}$collider Compact Linear Collider (CLIC) [45-48], that will operate with the center of mass energies $\sqrt{s}=380 \mathrm{GeV}, 1.4$ and $3 \mathrm{TeV}$. We first analyze the discovery reach of the doubly-charged Higgs boson at $380 \mathrm{GeV}$ center of mass energy. Subsequently, we carry out a detailed simulation for the very heavy doubly-charged Higgs boson with a mass around and beyond one TeV. For such a heavy Higgs, its final decay products are collimated, leading to fat-jets. We perform a jet-substructure analysis and tag the gauge bosons. We find that a heavy Higgs boson with a mass up to $1120 \mathrm{GeV}$ can be most optimally discovered with $5 \sigma$ significance at the $3 \mathrm{TeV}$ run of
CLIC with $95 \mathrm{fb}^{-1}$ of data. For lower masses, the range $160-172 \mathrm{GeV}$ can be covered with only $\mathcal{L} \sim 24 \mathrm{fb}^{-1}$ of luminosity. For the earlier discussions on Higgs triplet model at a linear collider, see [49-52]. For the other SM and BSM searches at CLIC and other linear colliders, see [47,53-67] for Higgs physics and effective field theory, [68-72] for different BSM scenarios, and [73-80] for seesaw and radiative neutrino mass model searches. For the discussion on probing dark-sector at $e^{+} e^{-}$collider, see $[81,82]$.

Our paper is organized as follows: we briefly review the basics of the Type-II seesaw model in Sec. II. In Sec. III, we discuss existing experimental constraints. In the subsequent subsections, Secs. IV A and IV B, we analyze in detail the production cross-sections and the discovery potential of the multi-jet final states at the $e^{+} e^{-}$collider. Finally, we present our conclusions in Sec. V.

\section{MODEL DESCRIPTION}

In addition to the SM Higgs field $\Phi$, the type-II seesaw model [10-13] contains an additional $S U(2)_{L}$ triplet Higgs field

$$
\Delta=\left(\begin{array}{cc}
\frac{\Delta^{+}}{\sqrt{2}} & \Delta^{++} \\
\Delta^{0} & -\frac{\Delta^{+}}{\sqrt{2}}
\end{array}\right) \sim(1,3,2) .
$$

We denote the neutral components of the SM doublet and triplet Higgs fields as $\Phi^{0}=\frac{1}{\sqrt{2}}\left(\phi^{0}+i \chi^{0}\right)$ and $\Delta^{0}=$ $\frac{1}{\sqrt{2}}\left(\delta^{0}+i \eta^{0}\right)$, respectively. The real scalars $\phi^{0}$ and $\delta^{0}$ acquire vevs denoted as $v_{\Phi}$ and $v_{\Delta}$ with $v^{2}=v_{\Phi}^{2}+v_{\Delta}^{2}=$ $(246 \mathrm{GeV})^{2}$. The light neutrino mass is proportional to the triplet vev $v_{\Delta}$. The new scalar field $\Delta$, being a triplet under $S U(2)$, interacts with the SM gauge bosons. The relevant kinetic term has the form

$$
\mathcal{L}_{\text {kin }}(\Delta)=\operatorname{Tr}\left[\left(D_{\mu} \Delta\right)^{\dagger}\left(D^{\mu} \Delta\right)\right],
$$

with the covariant derivative $D_{\mu} \Delta=\partial_{\mu} \Delta+i \frac{g}{2}\left[\tau^{a} W_{\mu}^{a}, \Delta\right]+$ $i g^{\prime} B_{\mu} \Delta$. The Yukawa interactions of $\Delta$ with the lepton fields are

$$
\mathcal{L}_{Y}(\Phi, \Delta)=Y_{\Delta} \overline{L_{L}^{c}} i \tau_{2} \Delta L_{L}+\text { H.c. }
$$

In the above, $Y_{\Delta}$ is a $3 \times 3$ matrix and $c$ denotes charge conjugation. The triplet field $\Delta$ carries lepton number +2 and hence the Yukawa term conserves lepton number. The scalar potential of the Higgs fields $\Phi$ and $\Delta$ is

$$
\begin{aligned}
V(\Phi, \Delta)= & m_{\Phi}^{2} \Phi^{\dagger} \Phi+\tilde{M}_{\Delta}^{2} \operatorname{Tr}\left(\Delta^{\dagger} \Delta\right)+\left(\mu \Phi^{T} i \tau_{2} \Delta^{\dagger} \Phi+\text { H.c. }\right) \\
& +\frac{\lambda}{4}\left(\Phi^{\dagger} \Phi\right)^{2}+\lambda_{1}\left(\Phi^{\dagger} \Phi\right) \operatorname{Tr}\left(\Delta^{\dagger} \Delta\right)+\lambda_{2}\left[\operatorname{Tr}\left(\Delta^{\dagger} \Delta\right)\right]^{2} \\
& +\lambda_{3} \operatorname{Tr}\left[\left(\Delta^{\dagger} \Delta\right)^{2}\right]+\lambda_{4} \Phi^{\dagger} \Delta \Delta^{\dagger} \Phi,
\end{aligned}
$$


where $m_{\Phi}$ and $\tilde{M}_{\Delta}$ are real parameters with mass dimension $1, \mu$ is the lepton-number violating parameter with positive mass dimension and $\lambda, \lambda_{1-4}$ are dimensionless quartic Higgs couplings.

There are seven physical Higgs states in mass basis, that arise after diagonalization of the scalar mass matrix written in the gauge basis. They are: the charged Higgs bosons $H^{ \pm \pm}, H^{ \pm}$, the neutral Higgs bosons $h^{0}, H^{0}$ and $A^{0}$. The two charged scalar fields $\Phi^{ \pm}$of $\Phi$ and $\Delta^{ \pm}$of $\Delta$ mix to give singly-charged states $H^{ \pm}$and the charged Goldstone $\chi^{ \pm}$ bosons. Similarly, the mixing between the two $C P$-odd fields $\left(\chi^{0}\right.$ and $\left.\eta^{0}\right)$ gives rise to $A^{0}$, and the neutral Goldstone boson $\rho^{0}$. Finally, we obtain the SM Higgs boson $(h)$ and a heavy Higgs boson $(H)$ via the mixing of the two neutral $C P$-even states $\Phi^{0}$ and $\delta^{0}$.

The physical masses of the doubly and singly charged Higgs bosons $H^{ \pm \pm}$and $H^{ \pm}$can be written as

$$
\begin{aligned}
& m_{H^{++}}^{2}=M_{\Delta}^{2}-v_{\Delta}^{2} \lambda_{3}-\frac{\lambda_{4}}{2} v_{\Phi}^{2}, \\
& m_{H^{+}}^{2}=\left(M_{\Delta}^{2}-\frac{\lambda_{4}}{4} v_{\Phi}^{2}\right)\left(1+\frac{2 v_{\Delta}^{2}}{v_{\Phi}^{2}}\right)
\end{aligned}
$$

The $C P$-even and $C P$-odd neutral Higgs bosons $h$, and $H$ have the physical masses

$$
\begin{aligned}
& m_{h}^{2}=\mathcal{T}_{11}^{2} \cos ^{2} \alpha+\mathcal{T}_{22}^{2} \sin ^{2} \alpha-\mathcal{T}_{12}^{2} \sin 2 \alpha, \\
& m_{H}^{2}=\mathcal{T}_{11}^{2} \sin ^{2} \alpha+\mathcal{T}_{22}^{2} \cos ^{2} \alpha+\mathcal{T}_{12}^{2} \sin 2 \alpha .
\end{aligned}
$$

In the above $\mathcal{T}_{11}, \mathcal{T}_{22}$ and $\mathcal{T}_{12}$ have the following expressions:

$$
\begin{aligned}
& \mathcal{T}_{11}^{2}=\frac{v_{\Phi}^{2} \lambda}{2}, \\
& \mathcal{T}_{22}^{2}=M_{\Delta}^{2}+2 v_{\Delta}^{2}\left(\lambda_{2}+\lambda_{3}\right), \\
& \mathcal{T}_{12}^{2}=-\frac{2 v_{\Delta}}{v_{\Phi}} M_{\Delta}^{2}+v_{\Phi} v_{\Delta}\left(\lambda_{1}+\lambda_{4}\right) .
\end{aligned}
$$

The $C P$-odd Higgs field $A^{0}$ has the mass term

$$
m_{A}^{2}=M_{\Delta}^{2}\left(1+\frac{4 v_{\Delta}^{2}}{v_{\Phi}^{2}}\right), \quad \text { with } \quad M_{\Delta}^{2}=\frac{v_{\Phi}^{2} \mu}{\sqrt{2} v_{\Delta}}
$$

The difference between $H^{ \pm \pm}$and $H^{ \pm}$masses is dictated by the coupling $\lambda_{4}$ of the scalar potential. For a positive $\lambda_{4}$, the $H^{ \pm \pm}$is lighter than $H^{ \pm}$. The mass difference $\Delta M^{2}$ is

$$
\Delta M^{2}=M_{H^{ \pm}}^{2}-M_{H^{ \pm \pm}}^{2} \sim \frac{\lambda_{4}}{2} v_{\Phi}^{2}+\mathcal{O}\left(v_{\Delta}^{2}\right) .
$$

Throughout our analysis, we consider the mass hierarchy $M_{H^{ \pm \pm}}<M_{H^{ \pm}}$. We have chosen $v_{\Delta}=0.1$. For this value of $v_{\Delta}$, the $H^{++}$decays to $W^{+} W^{+}$with $100 \%$ branching ratio.
The other Lagrangian parameters are chosen as $-\lambda_{1}=$ $\lambda_{2}=\lambda_{3}=\lambda_{4}=1.0, \lambda=0.52$. To change the $H^{++}$mass we vary $\mu$. Here we vary $\mu$ from 0.105 to 0.15 for $380 \mathrm{GeV}$ center of mass energy and from 1.56 to 4.65 for $3 \mathrm{TeV}$ center of mass energy.

Due to the nontrivial representations of $\Delta$, the Higgs triplet has interactions with a number of SM fermions and gauge bosons. This opens up a number of possible decay modes that can be explored at the LHC, and at future linear colliders. In the next section, we summarize the different direct experimental constraints on the doubly-charged Higgs boson mass and triplet vev.

\section{DECAY MODES AND EXPERIMENTAL CONSTRAINTS}

The most characteristic feature of the type II seesaw model is the presence of the doubly-charged Higgs boson $H^{ \pm \pm}$, that can decay into the leptonic or bosonic states and gives unique signatures at high energy colliders. The different decay modes and the branching ratios of the $H^{ \pm \pm}$ depend on the triplet vev $v_{\Delta}$. For smaller triplet vev, the $H^{ \pm \pm}$predominantly decays into the same-sign leptonic states $H^{ \pm \pm} \rightarrow l^{ \pm} l^{ \pm}$, whereas for larger $v_{\Delta}$, the gauge boson mode $H^{ \pm \pm} \rightarrow W^{ \pm} W^{ \pm}$becomes dominant [16,17]. The relevant decay widths are calculated to

$$
\begin{aligned}
& \Gamma\left(H^{ \pm \pm} \rightarrow l_{i}^{ \pm} l_{j}^{ \pm}\right)=\Gamma_{l_{i} l_{j}}=\frac{M_{H}^{ \pm \pm}}{\left(1+\delta_{i j}\right) 8 \pi}\left|\frac{M_{\nu_{i j}}}{v_{\Delta}}\right|^{2}, \\
& M_{\nu}=Y_{\Delta} v_{\Delta}, \\
& \Gamma\left(H^{ \pm \pm} \rightarrow W^{ \pm} W^{ \pm}\right) \\
& =\Gamma_{W^{ \pm} W^{ \pm}}=\frac{g^{2} v_{\Delta}^{2}}{8 \pi M_{H^{ \pm \pm}}} \sqrt{1-\frac{4}{r_{W}^{2}}}\left[\left(2+\left(r_{W} / 2-1\right)^{2}\right)\right] .
\end{aligned}
$$

In the above $M_{\nu}$ denotes the neutrino mass matrix, $i, j$ are the generation indices, $\Gamma_{l_{i} l_{j}}$ and $\Gamma_{W^{ \pm} W^{ \pm}}$are the partial decay widths for the $H^{ \pm \pm} \rightarrow l_{i}^{ \pm} l_{j}^{ \pm}$, and $H^{ \pm \pm} \rightarrow W^{ \pm} W^{ \pm}$channels, respectively. The parameter $r_{W}$ denotes the ratio of $H^{ \pm \pm}$ and the $W$ gauge boson masses, $r_{W}=\frac{M_{H^{ \pm \pm}}}{M_{W}}$. The branching fraction of the leptonic and bosonic mode becomes equal around the triplet vev $v_{\Delta} \sim 10^{-4} \mathrm{GeV}[16,17]$.

A number of searches have been proposed at the LHC to discover $H^{ \pm \pm}$using multilepton signatures. The searched modes in $[16-18,40]$ are pair and associated production with the $H^{ \pm \pm}$decaying into leptonic or gauge boson states. Below we discuss the existing constraints on $H^{ \pm \pm}$from LEP and LHC searches.

(i) Constraint from LEP-II: The search for doublycharged Higgs boson $H^{ \pm \pm}$decaying into charged 
leptons have been performed at LEP-II. This constrains the mass parameter $M_{H^{ \pm \pm}}>97.3 \mathrm{GeV}$ [44] at $95 \%$ C.L.

(ii) Constraints from pair and associated production: Stringent constraint on the $M_{H^{ \pm \pm}}$by analyzing $H^{ \pm \pm} \rightarrow l^{ \pm} l^{ \pm}$have been placed at the $13 \mathrm{TeV}$ LHC. The CMS collaboration looked for different leptonic flavors including $e e, e \mu, e \tau, \mu \mu, \mu \tau$ and $\tau \tau$. In addition, the CMS searches also include the associated production $p p \rightarrow H^{ \pm \pm} H^{\mp}$ and the subsequent decays, $H^{ \pm} \rightarrow l^{ \pm} \nu$. This combined channel of pair-production and associated production gives the stringent constraint $M_{H^{ \pm \pm}}>820 \mathrm{GeV}$ [34] at 95\% C.L for $e, \mu$ flavor. The constraint from ATLAS searches comes from pair-production. The bound is $M_{H^{ \pm \pm}}>870 \mathrm{GeV}$ at $95 \%$ C.L [33]. Note that these limits are valid only for a small triplet vev $v_{\Delta}<10^{-4} \mathrm{GeV}$.

(iii) Constraint from VBF: For larger values of the triplet vev $v_{\Delta} \geq 10^{-4} \mathrm{GeV}$, the leptonic branching ratio becomes smaller. Instead the decay mode $H^{ \pm \pm} \rightarrow$ $W^{ \pm} W^{ \pm}$is dominant. Therefore the searches in vector boson fusion (VBF) become more important. A search for $p p \rightarrow j j H^{ \pm \pm} \rightarrow j j W^{ \pm} W^{ \pm}$at the $8 \mathrm{TeV}$ LHC in the VBF channel sets a constraint on the triplet vev $v_{\Delta} \sim 25 \mathrm{GeV}$ for $M_{H^{ \pm \pm}} \sim 300 \mathrm{GeV}$ [35]. This constraint has been updated [36] using $13 \mathrm{TeV}$ data at the LHC.

Note that, for extremely small $v_{\Delta}$, the mass of the doubly-charged Higgs boson is very tightly constrained from pair-production searches. For a larger triplet vev, this constraint significantly relaxes. The VBF crosssection scales quadratically with the triplet vev and hence, increases for a very large vev. However, the range of $v_{\Delta} \sim 10^{-4}-10^{-1} \mathrm{GeV}$ cannot be probed at the $13 \mathrm{TeV}$ LHC in VBF channel, as the cross-section becomes extremely small in this range. Recently, in [41], the authors have looked for pair-production of $H^{ \pm \pm}$in large $v_{\Delta}$ region and analyzed the signature where the final state contains dilepton, multijet, and missing energy. The lighter mass $M_{H^{ \pm \pm}} \lesssim 190 \mathrm{GeV}$ can be probed at the $14 \mathrm{TeV}$ LHC with $3000 \mathrm{fb}^{-1}$ of data. In [39], the authors have used LHC $8 \mathrm{TeV}$ run-I result of same-sign dilepton to derive a bound $M_{H^{ \pm \pm}} \geq 84 \mathrm{GeV}$, relevant for large $v_{\Delta}$. For large mass of the doublycharged Higgs, the LHC cross section however becomes significantly smaller, as shown in Fig. 2. On the other hand, the fall in the cross-section at a $e^{+} e^{-}$collider is relatively smaller. This motivates us to explore the signatures of doubly-charged Higgs at a lepton collider, where the cross-section still remains larger for heavy charged Higgs masses. In the following sections, we explore the scope of a future linear collider to probe large $v_{\Delta}$ region with (a) a very low mass range of $H^{ \pm \pm}$, that is still experimentally allowed, and (b) a very heavy highly boosted $H^{ \pm \pm}$.

\section{LARGE TRIPLET VEV AND COLLIDER SIGNATURES}

In this section, we analyze the collider signatures of a doubly-charged Higgs boson at an $e^{+} e^{-}$collider and explore the sensitivity reach to probe low and high mass regimes. Throughout our analysis, we consider a large triplet vev $v_{\Delta} \geq 10^{-4} \mathrm{GeV}$, where the present experimental constraints are weak. As the prototype example, we consider CLIC [45-48] that will operate with three different center of mass energies $\sqrt{s}=380,1400 \mathrm{GeV}$ and $3 \mathrm{TeV}$. We present our simulation for $380 \mathrm{GeV}$ and $3 \mathrm{TeV}$ center of mass energies. The doubly-charged Higgs boson, $H^{ \pm \pm}$, can be produced at $e^{+} e^{-}$collider via photon and $Z$-boson mediated diagrams, as shown in Fig. 1. We show in Fig. 2 the respective production cross sections. As both of the diagrams are s-channel processes, the cross section reduces with increasing center-of-mass energy. For a relatively small center-of-mass energy $\sqrt{s}=$ $380 \mathrm{GeV}$, the maximum cross section reaches up to $\sigma \sim$ $506 \mathrm{fb}$ for $M_{H^{ \pm \pm}}=102 \mathrm{GeV}$. A rapid decline in the cross section occurs near $M_{H^{ \pm \pm}} \sim 190 \mathrm{GeV}$, close to kinematical threshold. For the choice of large $v_{\Delta}$, the produced particles $H^{ \pm \pm}$will decay into $W^{ \pm} W^{ \pm}$gauge bosons with almost $100 \%$ branching ratio. In the following, we will first discuss the low-mass regime, that can be probed in the $\sqrt{s}=380 \mathrm{GeV}$ run. Following that we discuss the high-mass regime, that can be explored at $3 \mathrm{TeV}$ center of mass energy and gives rise to specific signatures of boosted Higgs boson. In both cases we focus on multijet final states.

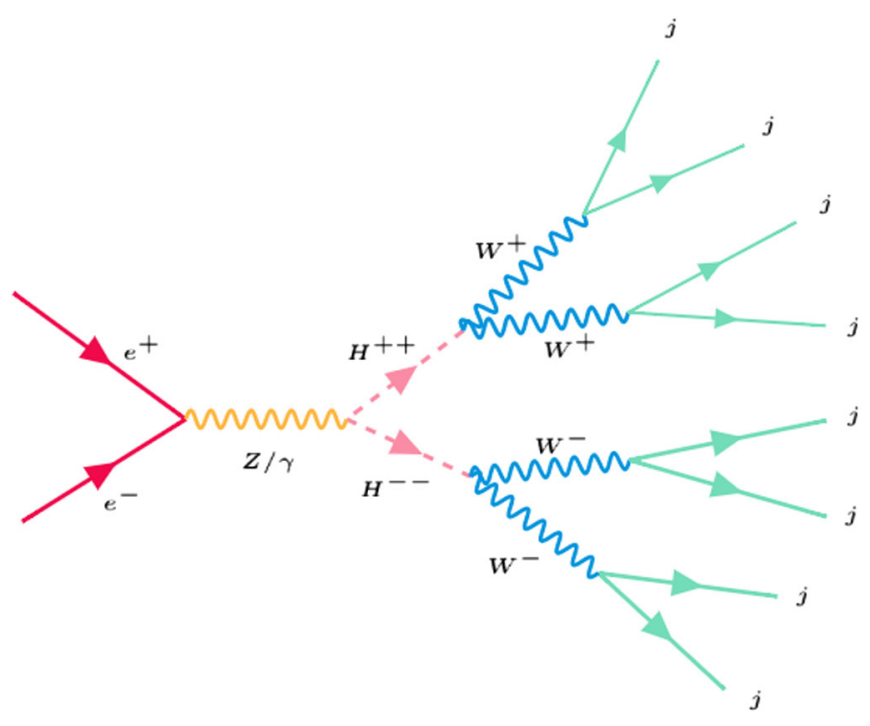

FIG. 1. The Feynman diagram for $H^{++} H^{--}$pair-production and its subsequent decays into gauge bosons. 


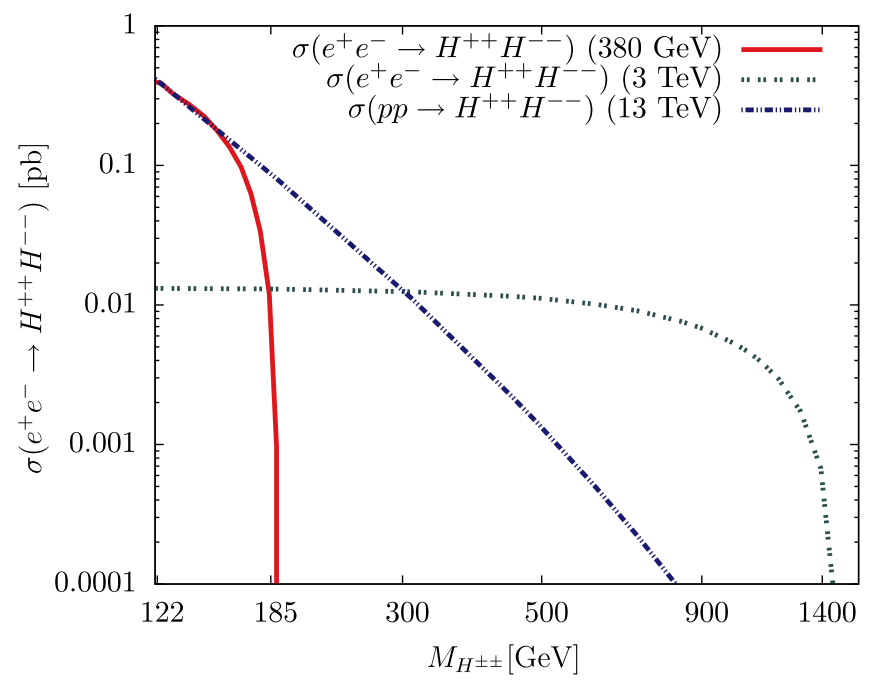

FIG. 2. The production cross-section at $e^{+} e^{-}$collider. The center of mass energies are $\sqrt{s}=380 \mathrm{GeV}$ and $3 \mathrm{TeV}$. For comparison, we also show the cross section at $13 \mathrm{TeV}$ LHC. The pair-production cross section increases by a factor of two, if CLIC uses $80 \%$, and $30 \%$ beam polarization for electron and positron beam.

\section{A. Low mass $\boldsymbol{H}^{ \pm \pm}$at $\sqrt{\boldsymbol{s}}=380 \mathrm{GeV}$}

We consider the pair-production of $H^{ \pm \pm}$, and its subsequent decay into $W^{ \pm} W^{ \pm}$at $\sqrt{s}=380 \mathrm{GeV}$. The produced $W^{ \pm}$decay dominantly into hadronic final states. Thus, to retain as much signal rate as possible, we focus on fully hadronic channel. Therefore, our model signature comprises of multijet events. In the subsequent analysis, we demand a high jet multiplicity, i.e., the number of jets $N_{\text {jet }} \geq 7$. For the signal, the production processes are

(i) $e^{+} e^{-} \rightarrow H^{ \pm \pm} H^{\mp \mp} \rightarrow 4 W \geq 7 j$ for $M_{H^{ \pm \pm}} \gtrsim 2 M_{W}$

(ii) $e^{+} e^{-} \rightarrow H^{ \pm \pm} H^{\mp \mp} \rightarrow W^{ \pm} j j W^{\mp} j j \geq 7 j$ for $M_{H^{ \pm \pm}}<$ $2 M_{W}$

In the former scenario the $H^{ \pm \pm}$decays predominantly into on-shell $W^{ \pm} W^{ \pm}$, while in the latter case $H^{ \pm \pm}$decays into one on-shell and one off-shell gauge bosons with subsequent decays into jets.

To simulate the events, we use first FeynRules [83] and generate the model file via Universal Feynrules Output (UFO) $[84,85]$. We compute the hard processes with the package MADGRAPH5_AMC@NLO [86], and pass the output (in LHE format) through PYTHIA 6 [87] for showering and hadronization. The detector simulation has been taken into account by DELPHES-3.3.0 [88], where we use the ILD card. Here we use anti- $k_{t}$ jet clustering algorithm [89] to form jets. Similar final states will be generated from a number of SM processes. We consider the following sets of backgrounds and perform a detailed simulation:

(i) $e^{+} e^{-} \rightarrow t \bar{t} \rightarrow 6 j$

(ii) $e^{+} e^{-} \rightarrow W^{+} W^{-}+3 j, W^{ \pm} \rightarrow 2 j$, and $e^{+} e^{-} \rightarrow Z Z+$ $3 j, Z \rightarrow 2 j$

(iii) $e^{+} e^{-} \rightarrow 7 j$

(iv) $e^{+} e^{-} \rightarrow W^{ \pm}+5 j, \quad W^{ \pm} \rightarrow j j, \quad$ and $\quad e^{+} e^{-} \rightarrow Z+$ $5 j, Z \rightarrow j j$

Among the backgrounds, $e^{+} e^{-} \rightarrow 7 j$ includes diagrams of coupling order $\alpha_{\mathrm{EW}}^{2} \alpha_{S}^{5}$ with quarks and gluons as intermediate particles. As listed above, we treat the $t \bar{t}$ and gauge boson mediated backgrounds separately. For the partonic event generation, we implement the following

TABLE I. The cross sections for the signal and background for the fully hadronic final states, arising from $e^{+} e^{-} \rightarrow H^{ \pm \pm} H^{\mp \mp} . \sigma_{p}$ refers to the partonic cross section. $\sigma_{d}$ is the cross section after taking into account detector effects. The last column represents the cross section with $b$-veto. The center-of-mass energy is $\sqrt{s}=380 \mathrm{GeV}$ and kinematic cuts are specified in the text.

\begin{tabular}{lccc}
\hline \hline & $e^{+} e^{-} \rightarrow H^{++} H^{--} \rightarrow N_{j} \geq 7 j$ & \\
\hline Mass $(\mathrm{GeV})$ & $\sigma_{p}(\mathrm{fb})$ & $\sigma_{d}\left(N_{j} \geq 7 j\right)(\mathrm{fb})$ & $\sigma_{d}\left(N_{j} \geq 7 j+b\right.$ veto $)(\mathrm{fb})$ \\
\hline 121 & 0.80 & 0.30 & 0.20 \\
137 & 2.08 & 0.94 & 0.66 \\
159 & 5.45 & 2.58 & 1.82 \\
172 & 5.04 & 2.48 & 0.38 \\
184 & 1.11 & 0.53 & 0.74 \\
\hline \hline
\end{tabular}

\begin{tabular}{lccc}
\hline \hline \multicolumn{3}{c}{ Backgrounds } \\
\hline Processes & $\sigma_{p}(\mathrm{fb}) \times 10^{-2}$ & $\sigma_{d}\left(N_{j} \geq 7 j\right)(\mathrm{fb}) \times 10^{-2}$ & $\sigma_{d}\left(N_{j} \geq 7 j+b\right.$ veto $)(\mathrm{fb}) \times 10^{-2}$ \\
\hline$e^{+} e^{-} \rightarrow t \bar{t} \rightarrow 6 j$ & 10341.0 & 338.0 & 36.0 \\
$W^{+} W^{-} 3 j, W^{ \pm} \rightarrow 2 j$ & 8.89 & 1.18 & 0.88 \\
$Z Z+3 j, Z \rightarrow 2 j$ & 0.98 & 0.13 & 0.10 \\
$7 j$ & 30.32 & 1.13 & 0.88 \\
$W^{ \pm}+5 j, W^{ \pm} \rightarrow j j$ & 30.18 & 4.64 & 3.54 \\
$Z+5 j, Z \rightarrow j j$ & 18.32 & 2.15 & 1.61 \\
\hline \hline
\end{tabular}


sets of cuts at MADGRAPH level both for the signal and backgrounds: the transverse momentum of light jets $p_{T}\left(j_{i}\right)>20 \mathrm{GeV}$ for all the final state partons, the pseudorapidity $|\eta|<5.0$, and the separation between the light jets $\Delta R\left(j_{i}, j_{j}\right)>0.4$.

We consider few illustrative mass points between $M_{H^{ \pm \pm}} \sim 121 \mathrm{GeV}$ and the kinematic threshold $M_{H^{ \pm \pm}} \sim$ $184 \mathrm{GeV}$, and display the signal cross sections in Table I. The cross sections $\sigma_{p}$ refers to the partonic cross section, while $\sigma_{d}$ is after taking into account reconstruction and detector effects. In addition to the cuts at the partonic level, we further implement few more selection cuts: the transverse momentum of jets $p_{T}\left(j_{i}\right)>20 \mathrm{GeV}$ for all the jets, pseudo-rapidity $|\eta|<4.5$ for jets, and the number of jets $N_{j} \geq 7 j$. The largest background arises from $t \bar{t} \rightarrow 6 j$, where the cross section is about $103 \mathrm{fb}$ at the partonic level. This is much larger than the largest signal cross section $5.45 \mathrm{fb}$, corresponding to $M_{H^{ \pm \pm}}=159 \mathrm{GeV}$. For other mass points, the ratio is even bigger. However, demanding high jet multiplicity $N_{j} \geq 7 j$ reduces this background to $\sigma_{d} \sim 3 \mathrm{fb}$. For the masses of the doublycharged Higgs boson $M_{H^{ \pm \pm}}=159$ and $172 \mathrm{GeV}$, the signal and background cross sections become almost equal after demanding higher jet multiplicity. A few comments are in order:

(i) Between the higher and lower mass ranges, i.e., $M_{H^{ \pm \pm}}>2 M_{W}$ and $M_{H^{ \pm \pm}}<2 M_{W}$, the former scenario corresponds to larger pair-production cross sections. The fall in cross section in the higher mass range occurs when $M_{H^{ \pm \pm}} \sim 184 \mathrm{GeV}$, where it approaches the kinematic threshold. For lower mass ranges, $M_{H^{ \pm \pm}} \sim 121 \mathrm{GeV}$, the reduction of crosssection after the detector effect occurs due to stronger kinematic cuts. The produced jets from a $H^{ \pm \pm}$with mass $M_{H^{ \pm \pm}} \sim 121 \mathrm{GeV}$ are often quite soft. With the constraint on jet transverse momentum $p_{T}>20 \mathrm{GeV}$ the reconstruction efficiency becomes smaller.

(ii) The signal comprises of hadronic final states with higher jet multiplicity. For the signal, $H^{ \pm \pm}$decays into two $W^{ \pm}$with subsequent decay into quarks, resulting in a final state with $N_{j}=8$. At a $e^{+} e^{-}$ collider, there are only a few SM processes that can generate a similar final state. A full reconstruction of the signal results in a fairly low reconstruction efficiency. Thus, we allow for one jet to be too soft or out of the kinematic cuts range.

In Table II, we derive the statistical significance $n_{s}=$ $\sigma_{d}(S) \sqrt{\mathcal{L}} / \sqrt{\sigma_{d}(S)+\sigma_{d}(B)}$ for our benchmark points corresponding to Table I. Here $\sigma_{d}(S)$ and $\sigma_{d}(B)$ represent the final cross-sections for the signal and background after all the selection cuts. Additionally, we also show the required luminosity to achieve a $5 \sigma$ significance. Other than the extreme low and high mass ranges $M_{H^{ \pm \pm}}=121$
TABLE II. The statistical significance $n_{s}$ for $\mathcal{L}=100 \mathrm{fb}^{-1}$. The third column displays the required luminosity to achieve $5 \sigma$ significance. The center-of-mass energy is $\sqrt{s}=380 \mathrm{GeV}$.

\begin{tabular}{lcr}
\hline \hline & $e^{+} e^{-} \rightarrow H^{++} H^{--} \rightarrow N_{j} \geq 7 j$ & \\
\hline Mass $(\mathrm{GeV})$ & $n_{s}$ & $\mathcal{L}\left(\mathrm{fb}^{-1}\right)$ \\
\hline 121 & 1.54 & 1054.14 \\
137 & 4.47 & 125.11 \\
159 & 10.48 & 22.76 \\
172 & 10.16 & 24.21 \\
184 & 2.65 & 355.99 \\
\hline \hline
\end{tabular}

TABLE III. The statistical significance $n_{s}(b)$ for $\mathcal{L}=100 \mathrm{fb}^{-1}$ and the required luminosity to achieve $5 \sigma$ significance, after implementing the $b$-veto. The center of mass energy is $\sqrt{s}=380 \mathrm{GeV}$.

\begin{tabular}{lrr}
\hline \hline \multicolumn{3}{c}{$e^{+} e^{-} \rightarrow H^{++} H^{--} \rightarrow N_{j} \geq 7 j+b$ veto } \\
\hline Mass $(\mathrm{GeV})$ & $n_{s}(b)$ & $\mathcal{L}\left(\mathrm{fb}^{-1}\right)$ \\
\hline 121 & 2.52 & 393.67 \\
137 & 6.32 & 62.59 \\
159 & 12.13 & 16.99 \\
172 & 11.81 & 17.92 \\
184 & 4.22 & 140.38 \\
\hline \hline
\end{tabular}

and $184 \mathrm{GeV}$, all other mass points have a large discovery prospect with $125 \mathrm{fb}^{-1}$ of data. In particular, we show that the doubly-charged Higgs boson with intermediate masses of $159 \mathrm{GeV}(172 \mathrm{GeV})$ can be discovered with $5 \sigma$ significance with only $\mathcal{L} \sim 22(24) \mathrm{fb}^{-1}$, respectively. From Table III, we can see that it further improves to $\mathcal{L} \sim$ $16(17) \mathrm{fb}^{-1}$ after applying a $b$-veto (50-60\% efficiency and $1 \%$ miss-tag efficiency), that helps in reducing the dominant top-quark pair background.

\section{B. Boosted heavy $\boldsymbol{H}^{ \pm \pm}$at $\sqrt{\boldsymbol{s}}=3 \mathrm{TeV}$}

We now consider heavy $H^{ \pm \pm}$with a mass $M_{H^{ \pm \pm}} \sim$ $1 \mathrm{TeV}$ and its decay into like-sign $W^{ \pm} W^{ \pm}$gauge bosons. The produced $W^{ \pm}$decays into hadronic as well as leptonic states. As before, we focus on the purely hadronic final states, which has the largest branching ratio. For such heavy $H^{ \pm \pm}$, each of the produced $W^{ \pm \pm}$boson will have large transverse momentum. For a $1.1 \mathrm{TeV} H^{ \pm \pm}$, their transverse momentum peaks around $p_{T} \sim 1 \mathrm{TeV}$, and most of the $W^{ \pm}$are produced in the central region. We show the transverse momentum, and the pseudo-rapidity distribution of $H^{ \pm \pm}$in Fig. 3, for the illustrative benchmark points $M_{H^{ \pm \pm}}=800 \mathrm{GeV}, 1120 \mathrm{GeV}$ and $1.4 \mathrm{TeV}$.

The final decay products of such heavy Higgs bosons are highly collimated, and can be reconstructed as fat-jets, see Fig. 4. Therefore, our model signature for such high mass $H^{ \pm \pm}$is

(i) $e^{+} e^{-} \rightarrow H^{ \pm \pm} H^{\mp \mp} \rightarrow W^{ \pm} W^{\mp} W^{ \pm} W^{\mp} \rightarrow 4$ fat - jet. 

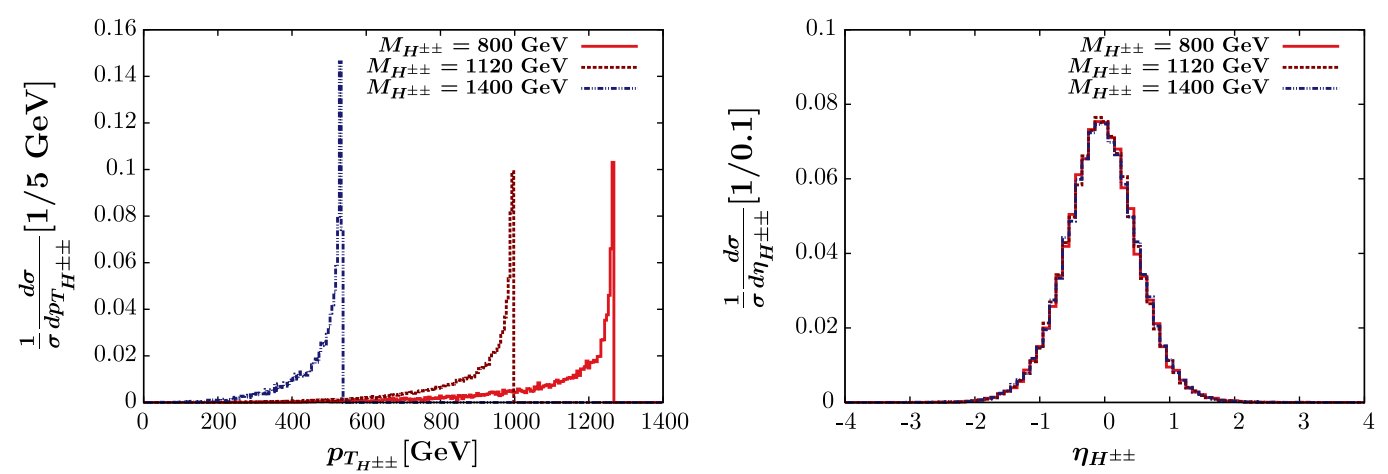

FIG. 3. The normalized distribution of the transverse momentum and the pseudorapidity for the produced $H^{ \pm \pm}$.

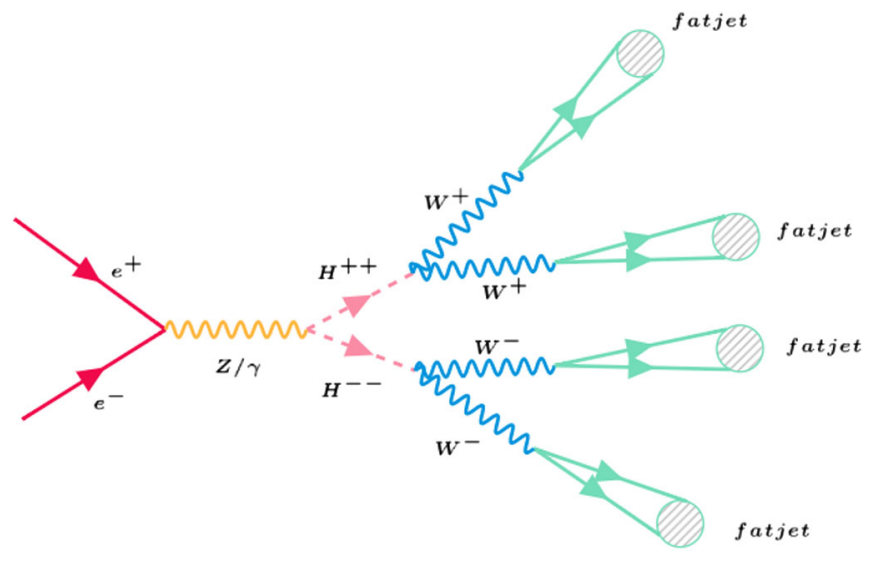

FIG. 4. The Feynman diagram for $H^{++} H^{--}$pair-production and its subsequent decays to 4 fat-jet.

To generate signal and backgrounds we use the same tool-chain as in Sec. IV A except the use of DELPHES. Here we analyze the output of PythiA8 [90] (in HepMC [91] format)and recluster fat-jets using Cambridge-Achen algorithm [92] in FASTJET-3.0.0 [93] with radius parameter $R=1.0$. In Fig. 5, we show the transverse momentum of the leading fat-jet $j_{1}$ and the 4 th leading fat-jet $j_{4}$. A number of backgrounds can lead to the final states with multiple fat-jets. These are: $4 j$ (includes both the QED and QCD contributions), $W^{+} W^{-} 2 j$, and $W^{+} / W^{-} 3 j$,
$W^{+} W^{-} Z j j$ and $t \bar{t}$, with subsequent decays of $W$ boson and the top quark into jets. The partonic cross sections of the signal and backgrounds are shown in Table IV. The cross-sections for $W^{+} W^{-} Z j j$ and $t \bar{t}$ are small compared to other backgrounds. Therefore, we do not include these backgrounds in our final analysis. Below we discuss in detail the preselection and selection cuts for the signal and backgrounds:

(i) Most of the signal events are in the central region with pseudorapidity distributed around $\eta_{H^{ \pm \pm}} \sim 0$, as can be seen in Fig. 3. Additionally, the signal jets have a very high $H_{T}$ (scalar sum of transverse momentum of all final state particles), as shown in Fig. 6. We consider no cuts on the signal at the parton level. While generating the backgrounds, we consider the following partonic cuts for $4 j$-the transverse momentum of the jets $p_{T}>60 \mathrm{GeV}$, and the jet-jet separation $\Delta R(j, j)>0.6$; for $W^{+} W^{-} 2 j\left(W^{ \pm}>2 j\right)$ and $W^{+} 3 j\left(W^{ \pm}>2 j\right)-p_{T}>$ $60 \mathrm{GeV}$ for the leading 4-jets, the transverse momentum $p_{T}>20 \mathrm{GeV}$ for the remaining jets, and the jet-jet separation $\Delta R(j, j)>0.4$. The $H_{T}$ and pseudo-rapidity cut is the same for all the backgrounds, $H_{T}>1000 \mathrm{GeV}$ and $|\eta|<2.5$. For $t \bar{t}$ samples we have put $\Delta R(b, j)>0.4$ separation cut and transverse momentum cut on leading two light jet as $p_{T}>60.0 \mathrm{GeV}$. Additionally, we also
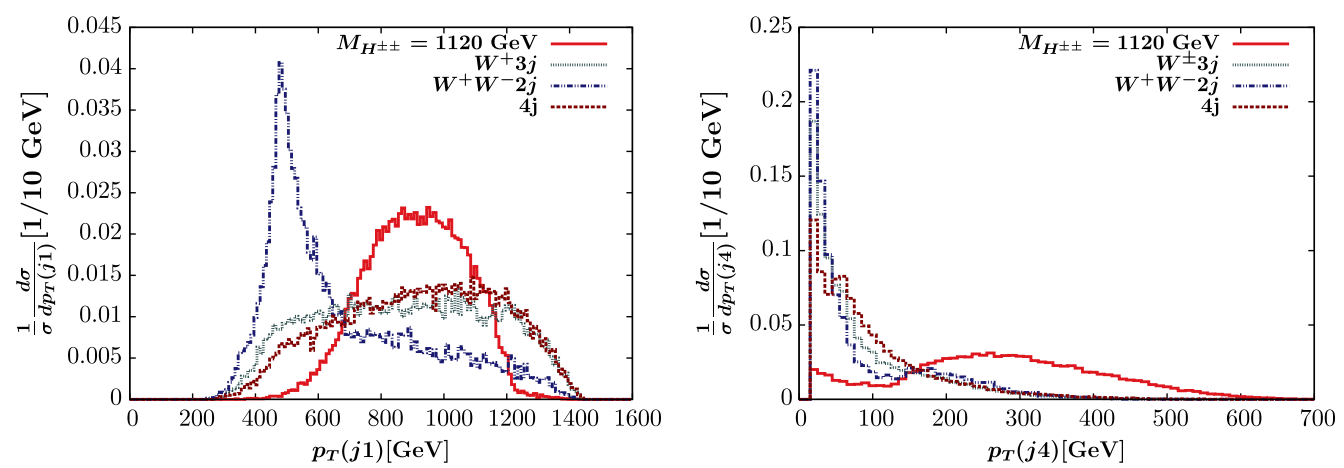

FIG. 5. The $p_{T}$ distribution of the leading and 4th leading fat-jets. For signal, we consider $M_{H^{ \pm \pm}}=1120 \mathrm{GeV}$. 
TABLE IV. The cut-flow for the signal and backgrounds. The cross sections are in fb. $\sigma_{p}$ refers to the partonic cross section. In the backgrounds the decays of the $W^{ \pm}$boson and top quark to jets are included. Here MD refers to mass-drop. See text for details.

\begin{tabular}{lccccccc}
\hline \hline \multicolumn{7}{c}{$e^{+} e^{-} \rightarrow H^{++} H^{--} \rightarrow W^{+} W^{+} W^{-} W^{-} \rightarrow N j_{\text {fat }}$} \\
\hline Masses $(\mathrm{GeV})$ & $\sigma_{p}(\mathrm{ab})$ & $4 j_{\text {fat }}(>120 \mathrm{GeV})$ & $4 \mathrm{MD}$ & 1 tagged & 2 tagged & 3-tagged & 4-tagged \\
\hline 800 & 1250 & 812.9 & 758.0 & 757.9 & 748.9 & 671.8 & 389.0 \\
1000 & 850.6 & 527.0 & 492.5 & 492.3 & 486.1 & 436.6 & 258.9 \\
1120 & 670.0 & 380.0 & 358.4 & 358.3 & 354.2 & 321.9 & 193.1 \\
1350 & 167.1 & 80.4 & 75.54 & 75.52 & 74.88 & 68.2 & 42.0 \\
1400 & 94.36 & 45.54 & 42.85 & 42.84 & 42.42 & 38.6 & 24.0 \\
\hline \hline
\end{tabular}

\begin{tabular}{lccccccc}
\hline \hline \multicolumn{7}{c}{ Backgrounds } \\
\hline Processes & $\sigma_{p}(\mathrm{ab})$ & $4 j(>120 \mathrm{GeV})$ & $4 \mathrm{MD}$ & 1 tagged & 2 tag ged & 3-tagged & 4-tagged \\
\hline $4 j$ & 6900.0 & 1310.0 & 895.0 & 360.0 & 68.0 & 5.5 & 0.0 \\
$W^{+} 3 j \& W^{-} 3 j$ & 1900.0 & 320.0 & 220.0 & 166.0 & 44.0 & 4.8 & $1.52 \times 10^{-1}$ \\
$W^{+} W^{-} 2 j$ & 190.0 & 25.6 & 17.7 & 15.6 & 8.3 & 1.23 & $5.7 \times 10^{-2}$ \\
$W^{+} W^{-} Z j j$ & 4.23 & $\ldots$ & $\ldots$ & $\ldots$ & $\ldots$ & $\ldots$ & $\ldots$ \\
$t \bar{t}$ & 42 & $\ldots$ & $\ldots$ & $\ldots$ & $\ldots$ & $\ldots$ & $\ldots$ \\
\hline \hline
\end{tabular}

demand $p_{T}$ of the bottom quarks more than $60 \mathrm{GeV}$ and the $p_{T}$ of the remaining light quarks more than $20 \mathrm{GeV}$.

(ii) The $\Delta R$ separation of the produced $W^{+} W^{+}, W^{+} W^{-}$ are shown in Fig. 7. It is evident that for relatively lower masses of $H^{ \pm \pm}$, such as $800 \mathrm{GeV}$, the $W^{+}$and $W^{+}$are closer, as compared to $1400 \mathrm{GeV}$. This occurs as the $H^{ \pm \pm}$with $800 \mathrm{GeV}$ mass is more boosted than the higher mass $H^{ \pm \pm}$. Hence, the produced $W^{ \pm} W^{ \pm}$are more collimated. For the separation between $W^{+}$and $W^{-}$this is opposite, i.e., for higher $\mathrm{H}^{++}$mass, the $W^{+}$and $W^{-}$separation is smaller as compared to the lower mass. When the mass of $\mathrm{H}^{++}$is large, the momentum of $\mathrm{H}^{++}$is relatively low. Therefore, the decay products of $\mathrm{H}^{++}$ are less collimated. Therefore in this scenario, there is a chance for overlap between jets from two W-bosons-one of them coming from $\mathrm{H}^{++}$and other coming from another $H^{--}$. This leads to smaller number of 4 fatjet events. From 2 nd column and 3rd column in Table IV, this is evident that after demanding four fatjet with $p_{T}>120 \mathrm{GeV}$ the drop in the cross section is larger for higher masses as compare to that of the lower masses.

(iii) The model signature contains four fat-jet with high momentum. We show in Fig. 5, the transverse momentum of leading and 4th leading fat-jet for $M_{H^{ \pm \pm}}=1120 \mathrm{GeV}$. Additionally, we also show the distributions of the backgrounds. It is evident that most of the jets have larger transverse momentum for signal, with $p_{T} \gg 100 \mathrm{GeV}$. Therefore, we design our selection cuts as (a) the number of fat-jets $N_{j_{\text {fat }}}=4$, (b) $p_{T_{j_{\text {fat }}}}>120 \mathrm{GeV}$ for all the fat-jets.

(iv) We further carry out substructure analysis for the fat-jets. To reconstruct the $W$ bosons we use the
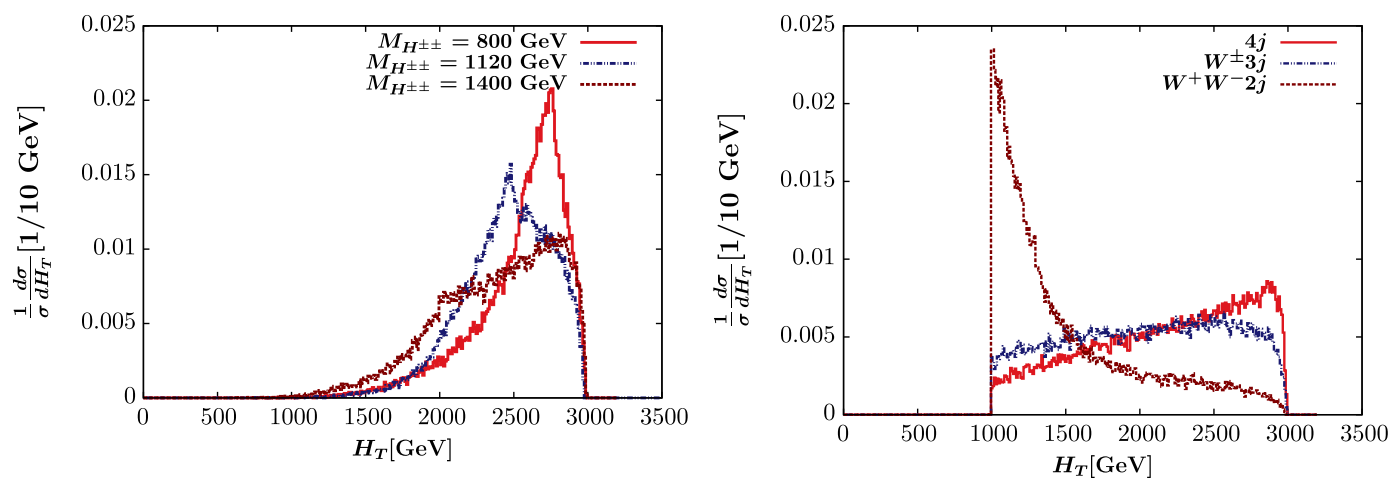

FIG. 6. The $H_{T}$ distribution of the jets at the partonic level. We consider three illustrative benchmark points $M_{H^{ \pm \pm}}=800,1120$, and $1400 \mathrm{GeV}$. 

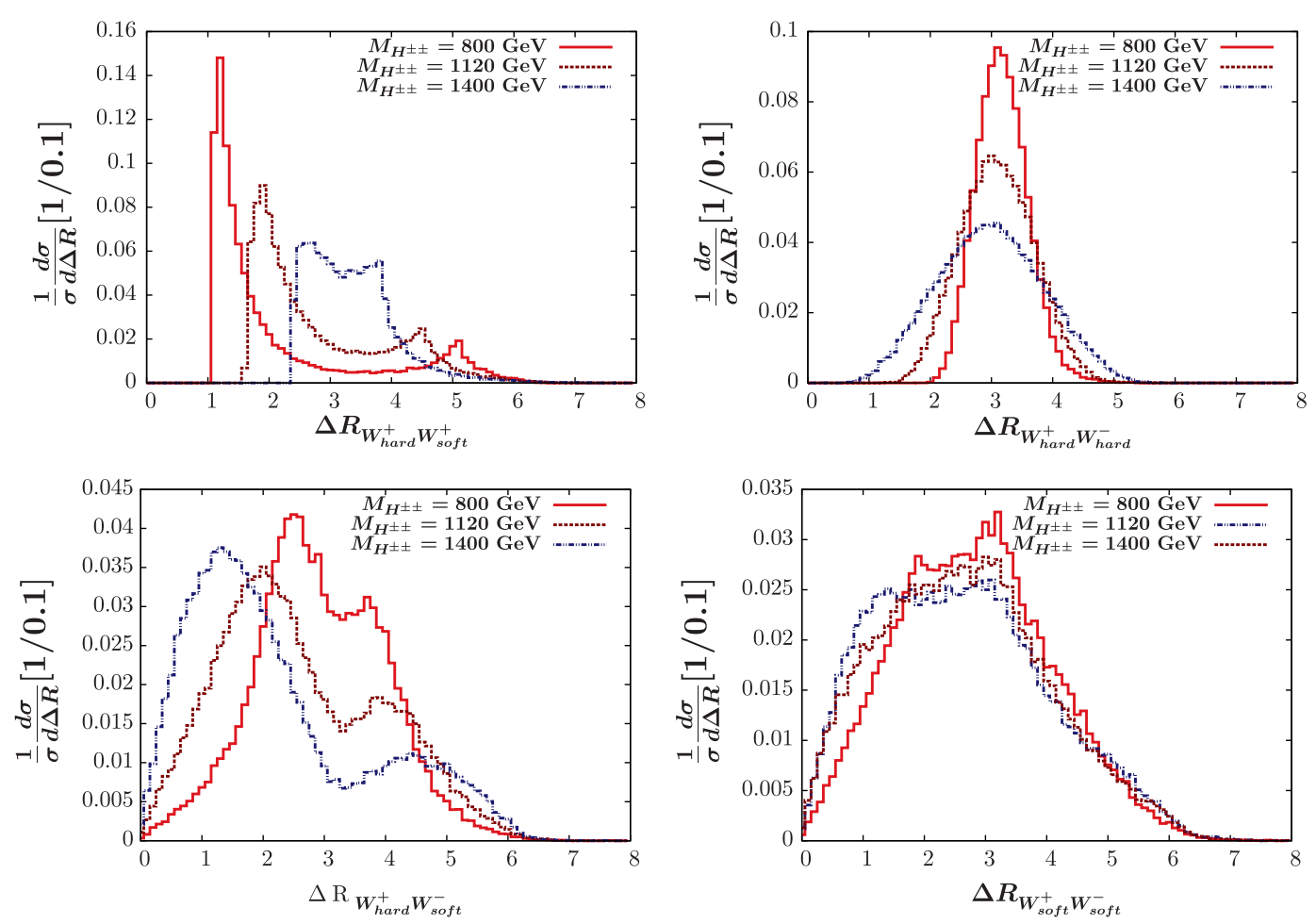

FIG. 7. The $\Delta R_{W W}$ distribution of the different $W^{ \pm}$, produced from the doubly charge Higgs $H^{ \pm \pm}$. The $W^{\prime}$ s in this figure are $p_{T}$ ordered.
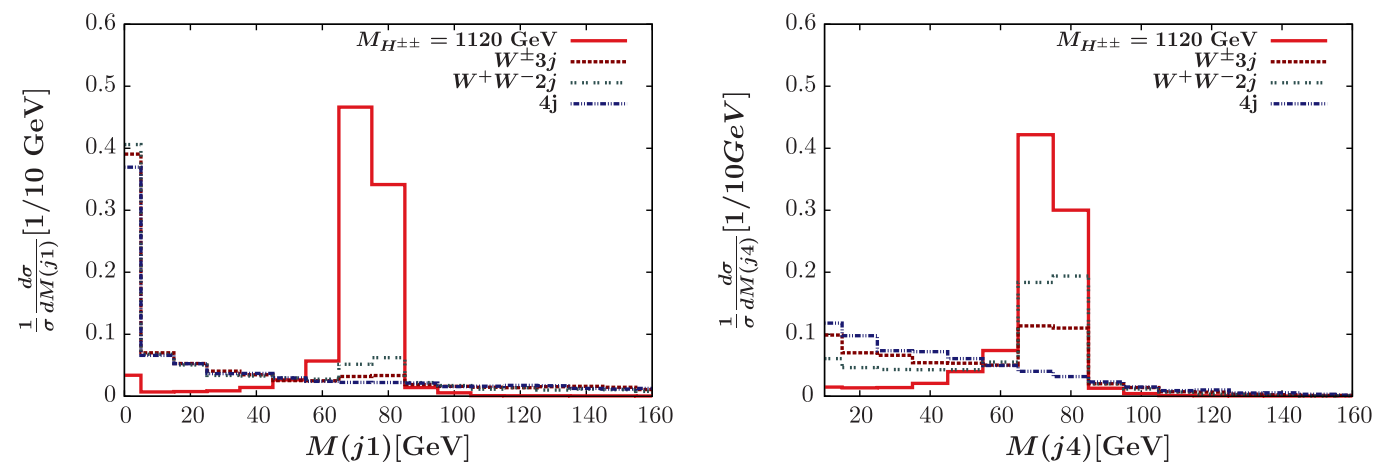

FIG. 8. The invariant mass of the fat-jet(leading and 4th leading) constructed using subjets four momentum. For signal, we consider $M_{H^{ \pm \pm}}=1120 \mathrm{GeV}$.

mass-drop tagger [94] of which compares the energy-sharings of subjets to indicate if the fat-jet was initiated by a $W$ boson or a parton. For the signal and background, we show the invariant mass of the two subjets inside the fat-jet in Fig. 8. For the signal, the subjets inside a fatjet are generated from the $W$. Therefore, the distribution peaks around the $W$ mass. For the different backgrounds, $4 j$ gives flat distribution, while $W^{+} W^{-} 2 j$ and $W^{+} 3 j$ shows smaller peak around $M_{W}$. As shown in Table IV, the backgrounds are significantly reduced after applying the selection cut $\left|M_{J_{1} J_{2}}-M_{W}\right| \leq 20 \mathrm{GeV}$. Here, $M_{J_{1} J_{2}}$ is the invariant mass of the subjets $J_{1}$ and $J_{2}$ inside a fat-jet. A detailed cut-flow chart is given in Table IV. If at least one fat-jet passes the invariant mass selection cut, we have 1-tagged event; if at least two fat-jet pass the cut, we have 2-tagged event and so on.

From Table IV, the effect of the substructure analysis is clearly evident. The largest background arises from the $e^{+} e^{-} \rightarrow 4 j$ events. At the partonic level we find a cross section of $\sigma_{p}(4 j) \sim 6.9 \mathrm{fb} \gg \sigma_{p}$ (signal). The higher transverse momentum cut on jet $p_{T}$ reduces the signal nominally, and the background by more than $\mathcal{O}(5)$ for $4 j, W W 2 j$ and $W+3 j$. Demanding that 4 fat-jets have a nontrivial substructure (referred to as mass-drop MD in Table IV) 
TABLE V. The statistical significance $n_{s}$ for $\mathcal{L}=500 \mathrm{fb}^{-1}$ and the required luminosity to achieve $5 \sigma$ significance. The c.m.energy is $\sqrt{s}=3 \mathrm{TeV}$. In the 2nd column, to derive significance, we consider 2 tagged events for $800-1120 \mathrm{GeV}$ mass range and 3 tagged events for the higher mass range. Here 2-tag implies two or more than two fat-jet masses are within the window of 60-100 GeV, and the fat-jets are tagged as $W$ jets. Similar criteria applies for 3-tagged jets.

\begin{tabular}{lcc}
\hline \hline & $e^{+} e^{-} \rightarrow H^{++} H^{--} \rightarrow W^{+} W^{+} W^{-} W^{-} \rightarrow N j_{\text {fat }}$ \\
\hline Masses & $n_{s}(2,3$-tagged & $\mathcal{L}\left(\mathrm{fb}^{-1}\right)$ (with \\
$(\mathrm{GeV})$ & $\left.\mathcal{L}=500 \mathrm{fb}^{-1}\right)$ & 2,3 -tagged) \\
\hline 800 & $17.96(2$-tag) & 38.75 \\
1000 & $13.95(2$-tag) & 64.23 \\
1120 & $11.49(2$-tag) & 94.68 \\
1350 & $5.40(3-$ tag) & 428.66 \\
1400 & $3.85(3$-tag) & 843.31 \\
\hline \hline
\end{tabular}

reduces the background even more. Finally, with the invariant mass cut for the subjets, all backgrounds become almost negligible. For the $H^{ \pm \pm}$masses between $800 \mathrm{GeV}$ to $1.1 \mathrm{TeV}$ one can achieve a $S / B \sim \mathcal{O}(10)$. We show the required luminosity to achieve a discovery in Table V. The 800-1120 GeV doubly-charged Higgs boson can be discovered with $39-95 \mathrm{fb}^{-1}$ of data with at least 2 fat-jet tagged as W-bosons. However, for higher masses, such as $1.4 \mathrm{TeV}$ a minimum 3 tagged jets will be required.

\section{DISCUSSION AND CONCLUSIONS}

The Type-II seesaw model consists of an extension of the scalar sector by a Higgs triplet field $\Delta$ with hypercharge $Y=+2$. The neutral component of the triplet acquires a vev and generates the light neutrino mass. One of the most attractive features of this model is the presence of the doubly-charged Higgs boson $H^{ \pm \pm}$. Depending on the triplet vev, $H^{ \pm \pm}$can decay into a number of final states, including same-sign leptons, same sign gauge bosons, and via cascade decay to three body final states. For the lower triplet vev where $H^{ \pm \pm} \rightarrow l^{ \pm} l^{ \pm}$decays are predominant, the doubly-charged Higgs boson mass is tightly constrained by LHC pair and associated production searches, $M_{H^{ \pm \pm}}>$ $820,870 \mathrm{GeV}$. However, the higher triplet vev region is poorly constrained by the VBF searches. Moreover, the LHC search is limited in the very high mass region $M_{H^{ \pm \pm}} \sim 1 \mathrm{TeV}$, where the cross section is tiny.

In this work, we consider an $e^{+} e^{-}$collider operating with two center-of-mass energies $\sqrt{s}=380 \mathrm{GeV}$ and $3 \mathrm{TeV}$, and probe the large $v_{\Delta}$ region $v_{\Delta} \geq 10^{-2} \mathrm{GeV}$.
We consider two mass regimes, (a) light $H^{ \pm \pm}$with mass $M_{H^{ \pm \pm}} \lesssim 180 \mathrm{GeV}$, and (b) a very heavy $H^{ \pm \pm}$with mass $M_{H^{ \pm \pm}} \sim 800-1400 \mathrm{GeV}$. We consider fully hadronic decays of the produced $W$ 's and perform a detailed analysis for the multi-jet final states.

For the $380 \mathrm{GeV}$ center of mass energy, we look into multijet final states with $N_{j} \geq 7 j$. We find that a doublycharged Higgs boson with mass $M_{H^{ \pm \pm}} \sim 160-172 \mathrm{GeV}$ can be discovered in the immediate run of the $e^{+} e^{-}$collider, with only integrated luminosity $\mathcal{L} \sim 24 \mathrm{fb}^{-1}$. This improves considerably once we apply a $b$-veto, reducing the $t \bar{t}$ background to $\sigma \sim \mathcal{O}(0.1) \mathrm{fb}$.

The higher mass range $M_{H^{ \pm \pm}} \geq 1 \mathrm{TeV}$ can be probed in the $\sqrt{s}=3 \mathrm{TeV}$ run of the $e^{+} e^{-}$collider. Note that, for such high masses of $H^{ \pm \pm}$the pair-production cross section at $13 \mathrm{TeV}$ LHC is significantly smaller. Therefore, an $e^{+} e^{-}$ collider with large center of mass energy is more suitable to probe the high mass range. For such heavy mass, the produced $W \mathrm{~s}$ are boosted and their subsequent decay products will be collimated, resulting in fat-jets. A number of SM processes, including $4 j, W^{ \pm} 3 j, W^{ \pm} W^{ \pm} 2 j$ can mimic the signal. To reduce backgrounds, we carry out a jetsubstructure analysis with $W$-tagging. We find that for the $800-1120 \mathrm{GeV}$ mass range, a minimum of two tagged jets can effectively reduce the total backgrounds to a level of $\sigma \sim \mathcal{O}(0.1) \mathrm{fb}$, whereas the signal cross section is $\sigma \sim \mathcal{O}(0.3-0.7) \mathrm{fb}$. For higher masses, three tagged jets are needed. A doubly-charged Higgs boson with mass between $800-1120 \mathrm{GeV}$ can be discovered with $\mathcal{L} \lesssim$ $95 \mathrm{fb}^{-1}$ of data. For even higher masses, such as $M_{H^{ \pm \pm}} \sim$ $1400 \mathrm{GeV}$, a discovery will require much higher integrated luminosities.

Thus, a future high-energy $e^{+} e^{-}$collider can provide an outstanding opportunity to probe weakly-coupled heavy particles, which are beyond the reach of the LHC.

\section{ACKNOWLEDGMENTS}

M. M. acknowledges the support of the DST-INSPIRE research Grant No. IFA14-PH-99. M. M. also thanks to the workshop 'Physics at CLIC', held at CERN, Geneva, where part of the work has been carried out. S. S. acknowledges the funding assistance provided by Royal Society International Exchange program and the hospitality of the Institute for Particle Physics Phenomenology (IPPP) at Durham University where part of the work has been carried out. We also acknowledge the use of SAMKHYA, the Institute of physics high performance computing facility. 
[1] S. Weinberg, Baryon and Lepton Nonconserving Processes, Phys. Rev. Lett. 43, 1566 (1979).

[2] F. Wilczek and A. Zee, Operator Analysis of Nucleon Decay, Phys. Rev. Lett. 43, 1571 (1979).

[3] P. Minkowski, $\mu \rightarrow e \gamma$ at a rate of one out of $10^{9}$ muon decays?, Phys. Lett. 67B, 421 (1977).

[4] R. N. Mohapatra and G. Senjanović, Neutrino Mass and Spontaneous Parity Violation, Phys. Rev. Lett. 44, 912 (1980).

[5] T. Yanagida, Horizontal symmetry and masses of neutrinos, Conf. Proc. C7902131, 95 (1979).

[6] M. Gell-Mann, P. Ramond, and R. Slansky, Complex spinors and unified theories, Conf. Proc. C790927, 315 (1979).

[7] J. Schechter and J. W. F. Valle, Neutrino masses in $\mathrm{SU}(2) \times$ U(1) theories, Phys. Rev. D 22, 2227 (1980).

[8] K. S. Babu, C. N. Leung, and J. T. Pantaleone, Renormalization of the neutrino mass operator, Phys. Lett. B 319, 191 (1993).

[9] S. Antusch, M. Drees, J. Kersten, M. Lindner, and M. Ratz, Neutrino mass operator renormalization in two Higgs doublet models and the MSSM, Phys. Lett. B 525, 130 (2002).

[10] M. Magg and C. Wetterich, Neutrino mass problem and gauge hierarchy, Phys. Lett. 94B, 61 (1980).

[11] T. P. Cheng and L.-F. Li, Neutrino masses, mixings and oscillations in $\mathrm{SU}(2) \times \mathrm{U}(1)$ models of electroweak interactions, Phys. Rev. D 22, 2860 (1980).

[12] G. Lazarides, Q. Shafi, and C. Wetterich, Proton lifetime and fermion masses in an SO(10) model, Nucl. Phys. B181, 287 (1981).

[13] R. N. Mohapatra and G. Senjanović, Neutrino masses and mixings in gauge models with spontaneous parity violation, Phys. Rev. D 23, 165 (1981).

[14] R. Foot, H. Lew, X. G. He, and G. C. Joshi, Seesaw neutrino masses induced by a triplet of leptons, Z. Phys. C 44, 441 (1989).

[15] A. G. Akeroyd and M. Aoki, Single and pair production of doubly charged Higgs bosons at hadron colliders, Phys. Rev. D 72, 035011 (2005).

[16] P. F. Perez, T. Han, G.-y. Huang, T. Li, and K. Wang, Neutrino masses and the CERN LHC: Testing type II seesaw, Phys. Rev. D 78, 015018 (2008).

[17] A. Melfo, M. Nemevsek, F. Nesti, G. Senjanović, and Y. Zhang, Type II seesaw at LHC: The roadmap, Phys. Rev. D 85, 055018 (2012).

[18] F. del Aguila and J. A. Aguilar-Saavedra, Distinguishing seesaw models at LHC with multi-lepton signals, Nucl. Phys. B813, 22 (2009).

[19] S. Chakrabarti, D. Choudhury, R. M. Godbole, and B. Mukhopadhyaya, Observing doubly charged Higgs bosons in photon-photon collisions, Phys. Lett. B 434, 347 (1998).

[20] M. Aoki, S. Kanemura, and K. Yagyu, Testing the Higgs triplet model with the mass difference at the LHC, Phys. Rev. D 85, 055007 (2012).

[21] A. G. Akeroyd and H. Sugiyama, Production of doubly charged scalars from the decay of singly charged scalars in the Higgs Triplet Model, Phys. Rev. D 84, 035010 (2011).
[22] E. J. Chun and P. Sharma, Search for a doubly-charged boson in four lepton final states in type II seesaw, Phys. Lett. B 728, 256 (2014).

[23] F. del Águila and M. Chala, LHC bounds on lepton number violation mediated by doubly and singly-charged scalars, J. High Energy Phys. 03 (2014) 027.

[24] J. Alcaide, M. Chala, and A. Santamaria, LHC signals of radiatively-induced neutrino masses and implications for the ZeeBabu model, Phys. Lett. B 779, 107 (2018).

[25] S. Banerjee, M. Frank, and S. K. Rai, Higgs data confronts sequential fourth generation fermions in the Higgs triplet model, Phys. Rev. D 89, 075005 (2014).

[26] Z. Kang, J. Li, T. Li, Y. Liu, and G.-Z. Ning, Light doubly charged Higgs boson via the $W W^{*}$ channel at LHC, Eur. Phys. J. C 75, 574 (2015).

[27] Z.-L. Han, R. Ding, and Y. Liao, LHC phenomenology of type II seesaw: Nondegenerate case, Phys. Rev. D 91, 093006 (2015).

[28] Z.-L. Han, R. Ding, and Y. Liao, LHC phenomenology of the type II seesaw mechanism: Observability of neutral scalars in the nondegenerate case, Phys. Rev. D 92, 033014 (2015).

[29] K. S. Babu and S. Jana, Probing doubly charged Higgs bosons at the LHC through photon initiated processes, Phys. Rev. D 95, 055020 (2017).

[30] A. Arhrib, R. Benbrik, M. Chabab, G. Moultaka, M. C. Peyranere, L. Rahili, and J. Ramadan, The Higgs potential in the type II seesaw model, Phys. Rev. D 84, 095005 (2011).

[31] P. S. Bhupal Dev, D. K. Ghosh, N. Okada, and I. Saha, $125 \mathrm{GeV}$ Higgs boson and the type-II seesaw model, J. High Energy Phys. 03 (2013) 150.

[32] D. Das and A. Santamaria, Updated scalar sector constraints in the Higgs triplet model, Phys. Rev. D 94, 015015 (2016).

[33] M. Aaboud et al. (ATLAS Collaboration), Search for doubly charged Higgs boson production in multi-lepton final states with the ATLAS detector using proton-proton collisions at $\sqrt{s}=13$ TeV, Eur. Phys. J. C 78, 199 (2018).

[34] CMS Collaboration, A search for doubly-charged Higgs boson production in three and four lepton final states at $\sqrt{s}=13 \mathrm{TeV}$, CERN Technical Report No. CMS-PASHIG-16-036, 2017.

[35] V. Khachatryan et al. (CMS Collaboration), Study of Vector Boson Scattering and Search for New Physics in Events with Two Same-Sign Leptons and Two Jets, Phys. Rev. Lett. 114, 051801 (2015).

[36] A. M. Sirunyan et al. (CMS Collaboration), Observation of Electroweak Production of Same-Sign W Boson Pairs in the Two Jet and Two Same-Sign Lepton Final State in ProtonProton Collisions at $\sqrt{s}=13 \mathrm{TeV}$, Phys. Rev. Lett. 120, 081801 (2018).

[37] S. Kanemura, K. Yagyu, and H. Yokoya, First constraint on the mass of doubly-charged Higgs bosons in the same-sign diboson decay scenario at the LHC, Phys. Lett. B 726, 316 (2013).

[38] S. Kanemura, M. Kikuchi, K. Yagyu, and H. Yokoya, Bounds on the mass of doubly-charged Higgs bosons in the same-sign diboson decay scenario, Phys. Rev. D 90, 115018 (2014). 
[39] S. Kanemura, M. Kikuchi, H. Yokoya, and K. Yagyu, LHC Run-I constraint on the mass of doubly charged Higgs bosons in the same-sign diboson decay scenario, Prog. Theor. Exp. Phys. (2015) 051B02.

[40] M. Mitra, S. Niyogi, and M. Spannowsky, Type-II seesaw and multilepton signatures at hadron colliders, Phys. Rev. D 95, 035042 (2017).

[41] D. K. Ghosh, N. Ghosh, I. Saha, and A. Shaw, Revisiting the high-scale validity of type-II seesaw model with novel LHC signature, Phys. Rev. D 97, 115022 (2018).

[42] C. Englert, P. Schichtel, and M. Spannowsky, Same-sign W pair production in composite Higgs models, Phys. Rev. D 95, 055002 (2017).

[43] C. Hays, M. Mitra, M. Spannowsky, and P. Waite, Prospects for new physics in $\tau \rightarrow l \mu \mu$ at current and future colliders, J. High Energy Phys. 05 (2017) 014.

[44] J. Abdallah et al. (DELPHI Collaboration), Search for doubly charged Higgs bosons at LEP-2, Phys. Lett. B 552, 127 (2003).

[45] E. Accomando et al. (CLIC Physics Working Group Collaboration), Physics at the CLIC multi-TeV linear collider, in Proceedings, 11th International Conference on Hadron spectroscopy (Hadron 2005): Rio de Janeiro, Brazil, 2005, 2004, arXiv:hep-ph/0412251.

[46] L. Linssen, A. Miyamoto, M. Stanitzki, and H. Weerts, Physics and detectors at CLIC: CLIC conceptual design report, CERN Yellow Report No. CERN-2012-003.

[47] H. Abramowicz et al. (CLIC Detector and Physics Study Collaboration), Physics at the CLIC $e^{+} e^{-}$Linear ColliderInput to the Snowmass process 2013, in Proceedings, 2013 Community Summer Study on the Future of U.S. Particle Physics: Snowmass on the Mississippi (CSS2013): Minneapolis, MN, USA, 2013, 2013, arXiv:1307.5288.

[48] N. Alipour Tehrani et al., CLICdet: The post-CDR CLIC detector model, Report No. CLICdp-Note-2017-001, 2017.

[49] J.-F. Shen and Z.-X. Li, Doubly charged Higgs bosons pair production through WW fusion at high-energy $e^{+} e^{-}$linear colliders, Europhys. Lett. 111, 31001 (2015).

[50] S. Blunier, G. Cottin, M. A. Díaz, and B. Koch, Phenomenology of a Higgs triplet model at future $e^{+} e^{-}$colliders, Phys. Rev. D 95, 075038 (2017).

[51] J. Cao and X.-Y. Tian, Doubly and singly charged Higgs pair production at high-energy $e^{+} e^{-}$linear colliders, Int. J. Mod. Phys. A 31, 1650056 (2016).

[52] Y.-C. Guo, C.-X. Yue, and Z.-C. Liu, The signatures of doubly charged leptons in future linear colliders, J. Phys. G 44, 085004 (2017).

[53] R. Contino, C. Grojean, D. Pappadopulo, R. Rattazzi, and A. Thamm, Strong Higgs interactions at a linear collider, J. High Energy Phys. 02 (2014) 006.

[54] S. Heinemeyer and C. Schappacher, Neutral Higgs boson production at $e^{+} e^{-}$colliders in the complex MSSM: A full one-loop analysis, Eur. Phys. J. C 76, 220 (2016).

[55] A. Thamm, R. Torre, and A. Wulzer, Future tests of Higgs compositeness: direct vs indirect, J. High Energy Phys. 07 (2015) 100.

[56] N. Craig, M. Farina, M. McCullough, and M. Perelstein, Precision Higgsstrahlung as a probe of new physics, J. High Energy Phys. 03 (2015) 146.
[57] G. Durieux, C. Grojean, J. Gu, and K. Wang, The leptonic future of the Higgs, J. High Energy Phys. 09 (2017) 014.

[58] J. Ellis, P. Roloff, V. Sanz, and T. You, Dimension-6 operator analysis of the CLIC sensitivity to new physics, J. High Energy Phys. 05 (2017) 096.

[59] R. M. Godbole, C. Hangst, M. Muhlleitner, S. D. Rindani, and P. Sharma, Model-independent analysis of Higgs spin and $C P$ properties in the process $e^{+} e^{-} \rightarrow t \bar{t} \Phi$, Eur. Phys. J. C 71, 1681 (2011).

[60] S. S. Biswal, R. M. Godbole, R. K. Singh, and D. Choudhury, Signatures of anomalous VVH interactions at a linear collider, Phys. Rev. D 73, 035001 (2006).

[61] D. Dannheim et al., CLIC e + e- linear collider studies, arXiv:1208.1402.

[62] B. Ananthanarayan, S. K. Garg, C. S. Kim, J. Lahiri, and P. Poulose, Top Yukawa coupling measurement with indefinite $C P$ Higgs in $e^{+} e^{-} \rightarrow \bar{t} \bar{t} \Phi$, Phys. Rev. D 90, 014016 (2014).

[63] M. Thomson, Model-independent measurement of the $\mathrm{e}^{+} \mathrm{e}^{-} \rightarrow \mathrm{HZ}$ cross section at a future $\mathrm{e}^{+} \mathrm{e}^{-}$linear collider using hadronic Z decays, Eur. Phys. J. C 76, 72 (2016).

[64] G. Milutinović-Dumbelović, I. Božović-Jelisavčić, C. Grefe, G. Kačarević, S. Lukić, M. Pandurović, P. Roloff, and I. Smiljanić, Physics potential for the measurement of $\sigma(H \nu \bar{\nu}) \times \mathrm{BR}\left(H \rightarrow \mu^{+} \mu^{-}\right)$at the $1.4 \mathrm{TeV}$ CLIC collider, Eur. Phys. J. C 75, 515 (2015).

[65] H. Wang and B. Yang, Top partner production at $e^{+} e^{-}$ collider in the littlest Higgs Model with $T$-parity, Adv. High Energy Phys. 2017, 5463128 (2017).

[66] H. Abramowicz et al., Higgs physics at the CLIC electronpositron linear collider, Eur. Phys. J. C 77, 475 (2017).

[67] S. Banerjee, B. Bhattacherjee, M. Mitra, and M. Spannowsky, The lepton flavour violating Higgs decays at the HL-LHC and the ILC, J. High Energy Phys. 07 (2016) 059.

[68] V. Ari, O. Çakir, and S. Kuday, Pair production of new heavy leptons with $U(1)^{\prime}$ charge at linear colliders, Int. J. Mod. Phys. A 29, 1450055 (2014).

[69] L. Xiao-Zhou, M. Wen-Gan, Z. Ren-You, and G. Lei, $W W \gamma / Z$ production in the Randall-Sundrum model at LHC and CLIC, Phys. Rev. D 87, 056008 (2013).

[70] C.-W. Chiang, S. Kanemura, and K. Yagyu, Phenomenology of the Georgi-Machacek model at future electronpositron colliders, Phys. Rev. D 93, 055002 (2016).

[71] S. Heinemeyer and C. Schappacher, Charged Higgs boson production at $e^{+} e^{-}$colliders in the complex MSSM: A full one-loop analysis, Eur. Phys. J. C 76, 535 (2016).

[72] A. Llamas-Bugarin, A. Gutiérrez-Rodríguez, and M. A. Hernández-Ruíz, Probing the electromagnetic dipole moments of the tau-neutrino in the $U(1)_{B-L}$ model at the ILC and CLIC energies, Phys. Rev. D 95, 116008 (2017).

[73] J. Barry, L. Dorame, and W. Rodejohann, Linear collider test of a neutrinoless double beta decay mechanism in leftright symmetric theories, Eur. Phys. J. C 72, 2023 (2012).

[74] A. Das and N. Okada, Inverse seesaw neutrino signatures at the LHC and ILC, Phys. Rev. D 88, 113001 (2013).

[75] S. Banerjee, P. S. B. Dev, A. Ibarra, T. Mandal, and M. Mitra, Prospects of heavy neutrino searches at future lepton colliders, Phys. Rev. D 92, 075002 (2015).

[76] S. Antusch, E. Cazzato, and O. Fischer, Sterile neutrino searches at future $e^{-} e^{+}, p p$, and $e^{-} p$ colliders, Int. J. Mod. Phys. A 32, 1750078 (2017). 
[77] A. Ahriche, S. Nasri, and R. Soualah, Radiative neutrino mass model at the $e^{-} e^{+}$linear collider, Phys. Rev. D 89, 095010 (2014).

[78] S. Antusch, E. Cazzato, and O. Fischer, Higgs production from sterile neutrinos at future lepton colliders, J. High Energy Phys. 04 (2016) 189.

[79] S. Antusch, E. Cazzato, and O. Fischer, Displaced vertex searches for sterile neutrinos at future lepton colliders, J. High Energy Phys. 12 (2016) 007.

[80] S. S. Biswal and P. S. B. Dev, Probing left-right seesaw models using beam polarization at an $e^{+} e^{-}$collider, Phys. Rev. D 95, 115031 (2017).

[81] Z. Chacko, Y. Cui, and S. Hong, Exploring a dark sector through the Higgs portal at a lepton collider, Phys. Lett. B 732, 75 (2014).

[82] J. R. Andersen, M. Rauch, and M. Spannowsky, Dark sector spectroscopy at the ILC, Eur. Phys. J. C 74, 2908 (2014).

[83] A. Alloul, N. D. Christensen, C. Degrande, C. Duhr, and B. Fuks, FeynRules 2.0 - A complete toolbox for tree-level phenomenology, Comput. Phys. Commun. 185, 2250 (2014).

[84] C. Degrande, C. Duhr, B. Fuks, D. Grellscheid, O. Mattelaer, and T. Reiter, UFO - The universal FeynRules output, Comput. Phys. Commun. 183, 1201 (2012).

[85] P. de Aquino, W. Link, F. Maltoni, O. Mattelaer, and T. Stelzer, ALOHA: Automatic libraries of helicity amplitudes for Feynman diagram computations, Comput. Phys. Commun. 183, 2254 (2012).
[86] J. Alwall, R. Frederix, S. Frixione, V. Hirschi, F. Maltoni, O. Mattelaer, H.-S. Shao, T. Stelzer, P. Torrielli, and M. Zaro, The automated computation of tree-level and next-toleading order differential cross sections, and their matching to parton shower simulations, J. High Energy Phys. 07 (2014) 079.

[87] T. Sjostrand, L. Lonnblad, and S. Mrenna, PYTHIA 6.2: Physics and manual, arXiv:hep-ph/0108264.

[88] J. de Favereau, C. Delaere, P. Demin, A. Giammanco, V. Lemaítre, A. Mertens, and M. Selvaggi (DELPHES 3 Collaboration), DELPHES 3, A modular framework for fast simulation of a generic collider experiment, J. High Energy Phys. 02 (2014) 057.

[89] M. Cacciari, G. P. Salam, and G. Soyez, The anti-k(t) jet clustering algorithm, J. High Energy Phys. 04 (2008) 063.

[90] T. Sjostrand, S. Mrenna, and P.Z. Skands, A brief introduction to PYTHIA 8.1, Comput. Phys. Commun. 178, 852 (2008).

[91] M. Dobbs and J. B. Hansen, The HepMC C++ Monte Carlo event record for high energy physics, Comput. Phys. Commun. 134, 41 (2001).

[92] Y. L. Dokshitzer, G. D. Leder, S. Moretti, and B. R. Webber, Better jet clustering algorithms, J. High Energy Phys. 08 (1997) 001.

[93] M. Cacciari, G. P. Salam, and G. Soyez, FastJet user manual, Eur. Phys. J. C 72, 1896 (2012).

[94] J. M. Butterworth, A. R. Davison, M. Rubin, and G. P. Salam, Jet Substructure as a New Higgs Search Channel at the LHC, Phys. Rev. Lett. 100, 242001 (2008). 